\title{
Expertise, Teleoperation, and Task Constraints Affect the Speed-Curvature-Torsion Power Law in RAMIS
}

\author{
Yarden Sharon*, Ilana Nisky ${ }^{\dagger}$ \\ Department of Biomedical Engineering and Zlotowski Center for Neuroscience \\ Ben-Gurion University of the Negev, P. O. Box 653, Beer-Sheva, Israel
}

\begin{abstract}
Quantitative characterization of surgical movements can improve the quality of patient care by informing the development of new training protocols for surgeons, and the design and control of surgical robots. Here, we focus on the relationship between the speed of movement and its geometry that was extensively studied in computational motor control. In three-dimensional movements, this relationship is defined by a family of speed-curvature-torsion power laws, such as the one-sixth power law. We present a novel characterization of open and teleoperated suturing movements using the speed-curvature-torsion power-law analysis. We fitted the gain and the exponents of this power law to suturing movements of participants with different levels of surgical experience in open (using sensorized forceps) and teleoperated (using the da Vinci Research Kit/da Vinci Surgical System) conditions from two different datasets. We found that expertise and teleoperation significantly affected the gain and exponents of the power law, and that there were large differences between different segments of movement. These results confirm that the relationship between the speed and geometry of surgical movements is indicative of surgical skill, open a new avenue for understanding the effect of teleoperation on the movements of surgeons, and lay the foundation for the development of new algorithms for automatic segmentation of surgical tasks.
\end{abstract}

Keywords: Robot-assisted surgery; computational motor control; physical human-robot interaction; teleoperation; one-sixth power law; two-thirds power law; surgical skill.

\section{Introduction}

Surgery is a complex motor task that requires planning and execution of accurate movements. Computational motor control studies aim to develop models that explain how the brain controls the movements of our body, adapts, and learns [1-6]. These models can facilitate the understanding of how surgeons control the movements of their hands and instruments [7]. Such understanding

Received 28 September 2017; Revised 11 May 2018; Accepted 23 May 2018; Published 9 July 2018. Published in JMRR Special Issue on Continuum, Compliant, Congnitive, and Collaborative Surgical Robots. Guest Editors: Elena De Momi, Ren Hongliang and Chao Liu

Email Addresses: *shayar@post.bgu.ac.il, †nisky@bgu.ac.il

NOTICE: Prior to using any material contained in this paper, the users are advised to consult with the individual paper author(s) regarding the material contained in this paper, including but not limited to, their specific design(s) and recommendation(s).

This is an Open Access article published by World Scientific Publishing Company. It is distributed under the terms of the Creative Commons Attribution 4.0 (CC-BY) License. Further distribution of this work is permitted, provided the original work is properly cited. may help to develop effective training protocols for new surgeons, to improve the control of teleoperated surgical robots and new surgical systems, and augment algorithms for segmentation of surgical tasks.

The skill of a surgeon influences the outcome of surgical procedures [8], and surgeons need many years of training and many cases to master surgery $[9,10]$. Therefore, great efforts are invested in improving the acquisition and evaluation of surgical skill. Today, surgical skill assessment is mostly subjective, and it is done by surgeons who observe and rate other surgeons using global rating scales such as Global Operative Assessment of Laparoscopic Skills (GOALS) and Objective Structured Assessments of Technical Skills (OSATS) [11, 12]. The disadvantages of such subjective assessment are high variability between different observers, and the lack of information available to the observers. Thus, it is important to develop data-driven [13-18] objective metrics for surgical skill that will describe surgical performance in many details. Examples of such objective measures that are used today are task completion time [19-21] and 
path length [22-24]. However, these metrics are not sufficient for an accurate skill assessment. Other recent metrics are based on movement smoothness [25, 26], energy $[27,28]$, contact forces and robot arm acceleration [29], and others. Further quantifying of the characteristics of surgical movements may be useful in the development of new objective metrics for surgical skill.

Quantitative characterization of surgical movements may also contribute to the design of surgical robots. In teleoperated robot-assisted minimally-invasive surgery (RAMIS), surgeons use robotic manipulators to control the movements of instruments that are inserted into the body of the patient via small incisions [30]. Teleoperated robot-assisted surgery offers many advantages over open surgery [31, 32]. However, it also has drawbacks, such as the absence of touch information [33], and the effects of the dynamics of the robot on the movement of the surgeon $[34,35]$. As a first step towards improving the design of surgical robots, and making RAMIS teleoperation more natural, it is important to quantify how teleoperation affects the performance of surgical tasks compared to open surgery. In this paper, we investigate how teleoperation affects the characteristics of movements.

It is very easy to record surgical simulation or RAMIS data for analysis, but the resulting datasets are very long, and therefore, may be difficult to parse. To analyze surgical movement data effectively, it is necessary to divide the complex surgical movements into simpler segments. Thus, developing algorithms for automatic segmentation of surgical tasks can contribute to surgical skill assessment [36] and other related fields. Previous studies used various machine learning algorithms for surgical task segmentation [37-40]. Segmentation of complex movements into smaller segments was also studied in human motor control using the spatiotemporal properties of movement, and specifically the relationship of movement speed and its geometry [41]. A similar approach is also useful for the segmentation of complex surgical tasks. Such segmentation will highlight segments that are distinct in terms of their movement coordination rather than contextual information.

With these three important applications in mind, we analyzed the relationship between the speed, curvature, and torsion of suturing movements. Planar curved movements are characterized by the two-thirds power law that relates the speed of motion to its curvature [42]. This power law was used for surgical motion analysis [43, 44]. However, it was recently proposed that the speed of threedimensional movements also depends on torsion [45, 46]. In this paper, we present a novel analysis of suturing movements using the three-dimensional speed-curvature-torsion power law, and its utility in characterizing the effects of expertise, teleoperation, and task segment.

To demonstrate this approach, we used two different datasets that focused on different aspects of surgical suturing. The first dataset was collected during teleoperated (using the da Vinci Research Kit (dVRK) [18]) and open suturing movements [47]. The second dataset is teleoperated suturing movements using the da Vinci Surgical System (dVSS, Intuitive Surgical, Inc., Sunnyvale, CA) [17] from the JHU-ISI Gesture and Skill Assessment Working Set (JIGSAWS) [48] - a surgical activity dataset for human motion modeling. We tested whether the speedcurvature-torsion power law describes the suturing movements better than the simpler speed-curvature power law. We then used the parameters of the speedcurvature-torsion power law to characterize the suturing movements. We hypothesized that the gain and exponents of the power law will depend on expertise level, teleoperation (open suturing compared to robot-assisted suturing), and segment (within the suturing task). In the next section, we present the background on the power laws that relate the speed of movement to its geometry, and our predictions about the effects of expertise, teleoperation, and segment.

\section{Spatiotemporal Power Laws}

The relationship between the speed of movement and its geometry was studied extensively. The two-thirds power law is often stipulated as [42]

$$
v=\alpha \kappa^{-\frac{1}{3}}
$$

where $v$ is the movement speed, $\alpha$ is a constant, called the speed gain factor, and $\kappa$ is the curvature of the path, which measures the local deviation from straightness [45]. This power law was documented in a variety of movements [49-52]. However, it was found that this law does not adequately describe three-dimensional movements [53], and instead, the one-sixth power law was proposed $[45,46]$ :

$$
v=\alpha \kappa^{-\frac{1}{3}}|\tau|^{-\frac{1}{6}}
$$

where $\alpha$ is constant and called the speed gain factor, $\kappa$ is the curvature, and $\tau$ is the torsion of the path, which measures the local deviation from planarity.

Several studies suggested that in various conditions, the values of the exponents may be other than the $-1 / 3$ and $-1 / 6$ that appear in Eqs. (1) and (2) while still preserving the speed-curvature [54] and speed-curvature-torsion power laws [46]. For example, an analysis of shape tracing revealed that the exponents of the speedcurvature-torsion power law vary between shapes [46]. We hypothesized that a similar analysis may be useful for characterizing suturing movements, and used regression for fitting the exponents of the power laws together with the speed gain factor, i.e.

$$
v=\alpha \kappa^{\beta},
$$

for the planar speed-curvature power law, and

$$
v=\alpha \kappa^{\beta}|\tau|^{\gamma},
$$


for the three-dimensional speed-curvature-torsion power law. Because suturing movements are threedimensional, we expected that the speed-curvaturetorsion law will describe the data better than the planar law. We used model comparison techniques to verify this. We also expected that expertise level, teleoperation condition, and movement segment will affect the values of the parameters of the speed-curvature-torsion power law.

The first effect that we examined is expertise level. The speed gain factor $\alpha$ is related to overall tempo of motion [55] (see Eqs. (3) and (4)). Previous studies showed that there are differences between completion times of participants with different surgical skill levels $[20,21,23]$. In addition, the speed gain factor $\alpha$ from the two-thirds power law was used to predict surgical skill [44]. Therefore, we expected that the speed gain factor $\alpha$ will increase with surgical expertise.

In addition, for drawing movements, it was previously found that the exponent of the two-thirds power law depends on age. For children, the exponent differs from $-1 / 3$, and it converges to the typical adult value with age [49]. However, it is not clear whether this change in the exponent is caused by the difference between the motor systems of children and adults, or by the learning of new motor tasks. In our study, some of the participants have no surgical experience, and therefore, the suturing task is completely novel to them. In contrast, some of the participants are experienced surgeons who are already familiar with the task. Therefore, we hypothesized that the exponent $\beta$ of the speed-curvature-torsion power law in suturing movements will decrease (i.e. increase in its absolute value) with surgical expertise.

The second effect we investigated is teleoperation. The dynamics of the surgical manipulators and the teleoperation controllers impose challenges and constraints on the motor control system of the surgeon; these factors affect user movements [34, 35]. In addition, a recent study showed that the exponent of the two-thirds power law (Eq. (1)) in water is significantly different than in air [56], suggesting that dynamic constraints affect the relationship between the speed and curvature in planar movements. Based on this result, we assumed that the teleoperation-induced constraints would be reflected as differences in the parameters of the speed-curvaturetorsion power law between the teleoperated and the open condition.

The last effect that we investigated is differences between different movement segments. Studies showed that movements in different shapes resulted in different values of exponents $[46,54]$. Based on these results, we hypothesized that different segments of the suturing movement will produce different values of exponents when fitting the data to the power-law model. In addition, previous studies showed that the speed gain factor of the two-thirds power law varies between different segments of movement, and may also help in movement segmentation. For example, this approach was used for segmentation of the complex movements in sign language [57], and for segmentation of surgical tasks $[43,58]$. Therefore, we expected that the values of the speed gain factor of the speed-curvature-torsion power law may also be used for segmentation of the suturing movements.

\section{Methods}

In this study, we analyzed two datasets. The first dataset, called here dVRK-Open, is the data that were collected in a previous study during teleoperated (using dVRK [18]) and open suturing movements [47]. The second dataset, called JIGSAWS, is teleoperated suturing movements (using dVSS [17], Intuitive Surgical, Inc., Sunnyvale, CA) from the JHU-ISI Gesture and Skill Assessment Working Set [48] - a surgical activity dataset for human motion modeling. In the following subsections, we first describe each dataset with its corresponding data analysis (Secs. 3.1 and 3.2), then we describe the fitting of the power laws (Sec. 3.3), and we finish with a summary of the statistical analysis (Sec. 3.4).

\subsection{Dataset 1: dVRK-open}

This dataset was collected at Stanford University, and consists of kinematic recordings of teleoperated (using the da Vinci Research Kit) and open (using a surgical needle driver equipped with magnetic pose trackers) unimanual suturing on a bench-top model. These data include recordings from six experienced surgeons (five urologists with more than 120 robotic cases and one general surgeon with more than 150 robotic cases, selfreported) and ten nonmedical participants (engineering graduate students). One nonmedical participant had extensive experience with the experimental setup, and therefore, was removed from the analysis. In addition, during the experiment of one of the experienced participants, there were problems with the data recording, and this participant was also removed from the analysis. Full details of the experimental setup and procedures for this dataset are reported in [47].

In the teleoperated session, the participants performed the task using dVRK. They sat in front of the master console, and the task board was mounted on the patient-side table such that its position was fixed relative to the cameras (Fig. 1(a)). In the open session, the participants used a standard surgical needle driver. To provide similar context to the teleoperated session, the participants also sat in front of dVRK, and the task board was mounted on the armrest of dVRK (Fig. 1(b)). Two magnetic pose trackers (trakSTAR, Ascension Technology 


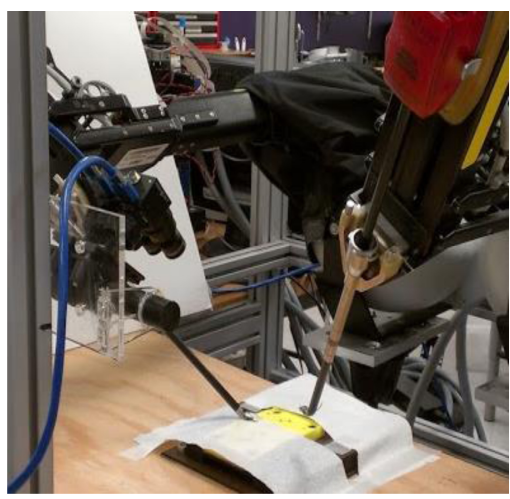

(a)

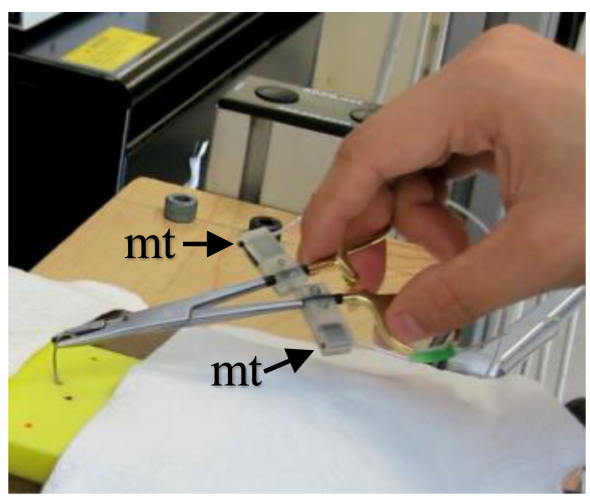

(b)

Fig. 1. The dVRK-Open experimental setups in the teleoperated and open suturing. (a) The task board and the instruments on the patient-side table in the teleoperated session. (b) A surgical needle driver with magnetic trackers ("mt") in the open session.

Corporation, Shelburne, VT) were mounted on the shafts of the needle driver, and their positions were recorded. To prevent signal distortion, the tracker was separated from the metal body of the driver by $2 \mathrm{~cm}$. We verified prior to the experiment that there was no metal between the trackers and the transmitter, and tested the integrity of the recorded signal using the Ascension Technology Corporation's proprietary software.

The task board was made of foam, and consisted of four marks (Fig. 2(a.III)): start (s), insertion (i), exit (e), and finish $(f)$. A single suturing trial included four segments (Fig. 2(a)): (I) transport, reaching with the head of the needle from $s$ to $i$; (II) insertion, driving the needle through the artificial tissue until its tip exits at $e$; (III) catching, opening the gripper and catching the tip of the needle; and (IV) extraction, pulling the needle and reaching to $f$ with its tail. The trial ended when the tail of the needle was placed at the end target, and left foot paddle or mouse button was pressed to indicate the trial end. In each session (teleoperated and open) each participant performed 80 trials, with breaks after every 10 trials. During the experiment, some of the trials were not performed according to the instructions, and some were not recorded properly. These trials were removed from the analysis. Among teleoperated sessions, 27 out of the 1120 trials of all the participants were removed, and in the open sessions, 51 out of the 1120 trials of all the participants were removed.

\subsubsection{Preprocessing}

In this study, we analyzed the Cartesian position of the needle driver. In the teleoperated session, we used the position of the right Patient Side Manipulator (PSM), which was recorded at $2 \mathrm{kHz}$. In the open session, the positions of the two magnetic pose trackers were recorded at $120 \mathrm{~Hz}$. We calculated the position of the needle driver tip using a transformation between the position of the trackers and the tip [59]. To find this transformation, we recorded a calibration dataset: we placed the driver's endpoint at a set location and recorded the position and orientation of the trackers over a large range of orientations of the needle driver. We used a linear regression over this calibration dataset to find the parameters of the transformation. Later, we interpolated and downsampled all the positions data (from both sessions) to $100 \mathrm{~Hz}$ using piecewise cubic Hermite interpolating polynomial (PCHIP).

\subsubsection{Segmentation}

We divided the movement into its stages (Fig. 2). Since there were no video recordings of the experiment, we built a segmentation algorithm that was based on the movement's trajectory and the opening angle of the needle driver. The transition between segments was determined by looking for remarkable points that met requirements that were specific to the structured suturing task of the dVRK-Open dataset. Importantly, these were not based on calculating spatiotemporal properties across the entire segment. Examples of such criteria are: a minimum of the velocity signal or a threshold of the

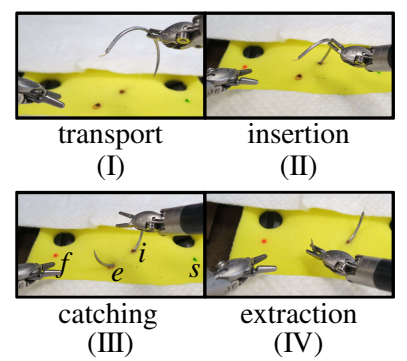

(a)

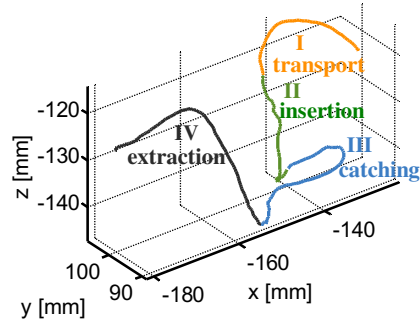

(b)
Fig. 2. The dVRK-Open dataset task segments. (a) The task board and task segments: (I) transport, (II) insertion, (III) catching, and (IV) extraction. (b) An example of the path of the right instrument. The numbers and the different colors indicate the four segments of the task. 
opening angle of the tool. This automatic segmentation was intended for simplifying the manual segmentation, and all the trials of all participants were checked manually and corrected if needed. In the teleoperated condition, five trials were segmented manually. In the open condition, 57 trials were segmented manually. Full details of the segmentation process are reported in [60]. The movement's trajectory and the opening angle were useful for segmenting the two first segments, until the participant opened the instrument for the first time. However, after the end of segment (II), there was substantial variability between the movements, and we could not use these kinematic properties for segmenting this part of the movement. For example, if the participant could not pull the needle out, and tried to insert the needle again, we cannot identify this on the basis of the kinematic properties. Hence, in this dataset, we focused only on the first and second segments.

\subsection{Dataset 2: JIGSAWS}

In the second part of our study, we used the JHU-ISI Gesture and Skill Assessment Working Set [48]. This dataset was collected using dVSS, and consists of kinematic and video recordings of elementary surgical tasks on a bench-top model. In this study, we used the kinematic data that was recorded during the suturing task. These data include recordings from eight surgeons with different robotic surgical experiences (four surgeons with less than $10 \mathrm{~h}$, two surgeons with $10-100 \mathrm{~h}$, and two surgeons with more than $100 \mathrm{~h}$, self-reported).

In each trial of the suturing task, the surgeon picked up a needle, reached the incision mark, and passed the needle via the mock tissue between two marked targets. After the needle pass, the surgeon used the left tool to extract the needle from the tissue, and passed it to the right tool. Then, the surgeon repeated the needle pass three more times. The mock tissue was made of elastic fabric. While performing the task, the surgeon was not allowed to move the camera or apply the clutch. Each surgeon repeats the suturing task five times. The JIGSAWS includes 39 trials of the suturing task. In our analysis we used the Cartesian positions of the PSMs that were recorded at $30 \mathrm{~Hz}$.

\subsubsection{Segmentation}

The JIGSAWS includes a manual segmentation of each movement into surgical gestures [48]. This segmentation was done by an individual who watched the video and in consultation with a surgeon. We used this segmentation in our analysis. In this paper, we will refer to each surgical gesture as a segment. In the suturing task, there were ten segments. However, some of the segments were not observed in all the trials, and some of the segments

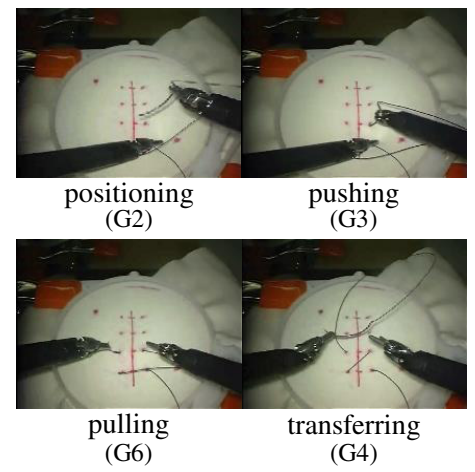

(a)

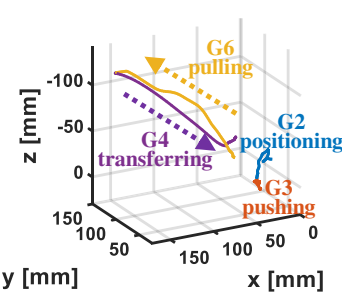

(b)
Fig. 3. JIGSAWS dataset task segments [48]. (a) The task workspace and an example of the task segments: (G2) positioning, (G3) pushing, (G6) pulling, and (G4) transferring. (b) An example of the path of the instruments from one suturing. The numbers and the different colors indicate the four segments of the task.

included movement of both tools (right and left). Therefore, we chose to analyze only four segments (Fig. 3): (G2) positioning, positioning the needle; (G3) pushing, pushing the needle through the tissue; (G6) pulling, pulling the suture with left hand; and (G4) transferring, transferring the needle from left to right.

\subsubsection{Surgical skill annotation}

The JIGSAWS dataset also includes a manual annotation of surgical technical skill [48]. Each trial in the JIGSAWS has a global rating score (GRS) that was assigned by an experienced surgeon who watched the videos. To assign the GRS, the surgeon used a modified OSATS method [12]. The GRS ranges from 6 (low technical skill) to 30 (high technical skill). For more information on the components of the GRS, see [48].

\section{3. $\quad$ Fitting the spatiotemporal power laws}

For each movement, we fitted two spatiotemporal power laws - a two-dimensional speed-curvature power law and a three-dimensional speed-curvature-torsion power law. In this subsection, we will describe the procedure of extracting the parameters of the spatiotemporal power laws from the data. First, we filtered the position data offline at $6 \mathrm{~Hz}$ with a second-order zero-lag Butterworth filter [Matlab's filtfilt()]. Then, we used the filtered position $\boldsymbol{x}(t)=[x(t), y(t), z(t)]^{T}$ to calculate the first, second, and third time derivatives of the position $[\dot{\boldsymbol{x}}(t)$, $\ddot{\boldsymbol{x}}(t)$, and $\dddot{\boldsymbol{x}}(t)$, respectively] using numerical differentiation. We filtered each derivative before calculating the next order derivative. For the derivatives, we used a second-order zero-lag low-pass Butterworth filter with a cutoff at $10 \mathrm{~Hz}$. 
To fit the power laws, we first calculated the speed $v$, the curvature $\kappa$, and the torsion $\tau$ for each sampling point. The speed was calculated as

$$
v(t)=\|\dot{\boldsymbol{x}}(t)\|=\sqrt{\dot{x}(t)^{2}+\dot{y}(t)^{2}+\dot{z}(t)^{2}},
$$

the curvature of the path was calculated as in [53]:

$$
\kappa(t)=\sqrt{\frac{\|\dot{\boldsymbol{x}}(t)\|^{2}|| \ddot{\boldsymbol{x}}(t) \|^{2}-\left(\dot{\boldsymbol{x}}(t)^{T} \ddot{\boldsymbol{x}}(t)\right)^{2}}{\|\dot{\boldsymbol{x}}(t)\|^{6}}},
$$

and the torsion was calculated as in [46]:

$$
\tau(t)=\frac{\dot{\boldsymbol{x}}(t) \bullet(\ddot{\boldsymbol{x}}(t) \times \dddot{\boldsymbol{x}}(t))}{\|\dot{\boldsymbol{x}}(t) \times \ddot{\boldsymbol{x}}(t)\|^{2}},
$$

where the operator - denotes a dot product, and the operator $\times$ denotes cross product. To fit the power laws, we took the log of both sides of Eqs. (3) and (4) to get

$$
\log v=\log (\alpha)+\beta \log (\kappa)
$$

and

$$
\log v=\log (\alpha)+\beta \log (\kappa)+\gamma \log (|\tau|) .
$$

Then, for each segment in each trial, using the $v, \kappa$, and $\tau$ that were calculated from the sampled data, we fitted linear regression models to find the values of the logarithm of the speed gain factor $[\log (\alpha)]$ and the exponents $\beta$ and $\gamma$.

In some of the movements, there were small parts in which the movement was planar. In these parts, it is not possible to fit the three-dimensional power law. To deal with this problem, we only used data points for which the absolute value of the torsion was higher than a $2-\mathrm{m}^{-1}$ threshold [46]. In total, $0.3 \%$ of the sampling points were below the threshold.

\subsection{Statistical analysis}

To compare between the two power laws, we used two model selection approaches. We tested whether adding the torsion as an independent variable significantly improved the quality of fit using an $F$-test for the incremental sum of squares [61]. In this test, we calculated the $p$-value for each segment, and if it was smaller than 0.05 , the decision was that the speed-curvaturetorsion power law is better for describing the data in this segment.

In addition, we calculated the Bayesian Information Criterion (BIC) [62] of the speed-curvature model $\left(\mathrm{BIC}_{2 \mathrm{D}}\right)$ and the speed-curvature-torsion model $\left(\mathrm{BIC}_{3 \mathrm{D}}\right)$. For each segment we compared the BIC values that are obtained from the two models:

$$
\Delta \mathrm{BIC}=\mathrm{BIC}_{2 \mathrm{D}}-\mathrm{BIC}_{3 \mathrm{D}} .
$$

If $\triangle \mathrm{BIC}$ was positive, the decision was that the threedimensional power law is better for describing the data in this segment. We also performed a $t$-test to check whether $\triangle \mathrm{BIC}$ is significantly greater than zero.

We then continued with an analysis of the speedcurvature-torsion power law. Each of the datasets was collected using different experimental procedures, and each had its own conditions and features. For example, only the dVRK-Open had an open condition, and only the JIGSAWS had GRS evaluation. Therefore, for each dataset, we performed a different analysis, and therefore, we describe the different statistical analyses separately for each dataset.

The statistical tests were performed using the Matlab Statistics Toolbox. In all the tests, statistical significance was determined at the 0.05 threshold. We used the Lilliefors test for the assumption of normality. Where needed, we used Mauchly's test for the assumption of sphericity, and none of our models violated sphericity. We used $t$-tests with Bonferroni correction for post-hoc multiple comparisons; the Bonferroni-corrected $p$-values are denoted as $p_{B}$, and are obtained by the multiplication of the original $p$-values with the number of comparisons in the family of tests.

\subsection{1. dVRK-Open}

We wanted to evaluate how expertise, teleoperation, and movement segment affect each of the power-law parameters $(\alpha, \beta, \gamma)$. For each of these parameters, separately for each participant, segment, and teleoperation condition, we calculated the average value over the entire 80 trials. Our experimental design was mixed each participant belonged only to one expertise group (experienced surgeon or nonmedical user), but performed all the segments in both conditions. Therefore, we fitted a three-way mixed-model ANOVA with the average value across trials of the parameter as the dependent variable. The independent variables were: expertise (experienced surgeon/nonmedical user, between participants), teleoperation condition (teleoperated/ open, within participants), segment [(I) transport/(II) insertion, within participants], and their first- and second-order interactions.

\subsubsection{JIGSAWS}

For this dataset, we performed several statistical analyses. We first tested whether there is a correlation between the parameters of the power law $(\alpha, \beta, \gamma)$ and surgical expertise, as measured by the GRS. To examine the existence of such correlation, we used regression analysis. Each participant performed five trials that consisted of several suturing movements, and hence, each trial had several repetitions of the different 
segments. We first calculated the average values of the parameter for the four segments in the trial. Then, using the average values of the four segments, we calculated one average value for each of the five trials. Then, we fitted regression models to this average as a function of the trial's GRS.

When fitting such regression models, two questions can be answered: the first one is whether there is a global effect of GRS on the power-law parameters. This effect would be mainly affected by the difference in expertise between the surgeons. To answer this question, we fitted a linear regression model with the trial's average value of the parameter as the dependent variable and the trial's GRS as the independent variable. The second question is whether within the different trials of each surgeon, there is a finer effect of the GRS on the power-law parameters. To answer this question, we fitted a one-way repeated-measures regression model with the trial's average value of the parameter as the dependent variable and the trial's GRS as the independent variable. This model still fits a single slope for all the participants, but a different intercept is fitted to the data of each participant. We chose not to include in this analysis an interaction term between participant and GRS because of the limited number of data points for each participant.

We were also interested in the question of how movement segment affects the parameters of the power law. Therefore, for each parameter $(\alpha, \beta, \gamma)$, we calculated the average value across all the repetitions of each segment for each participant. In other words, for each participant, we calculated four values for each parameter. Then, we fitted a one-way repeated-measures ANOVA model with the average value of the parameter as the dependent variable. The independent variable was segment ((G2) positioning/(G3) pushing/(G6) pulling/(G4) transferring, within participant).

\section{Results}

Overall, the participants of both datasets managed to complete the suturing task. In the dVRK-Open dataset, the average ( \pm 1 standard deviation) completion time of trial was $17.12 \pm 9.36 \mathrm{~s}$ in the teleoperated condition, and $8.12 \pm 4.38 \mathrm{~s}$ in the open condition. In the JIGSAWS dataset, the average completion time of trial was $110.36 \pm 43.27 \mathrm{~s}$. For more information on performance metrics, see [47, 60] for the dVRK-Open dataset and [63] for the JIGSAWS dataset.

To compare the speed-curvature and speed-curvature-torsion power laws, we calculated the $F$-test for the incremental sum of squares and the difference between the BICs of the two models. Our analysis showed that for $99.86 \%$ of the segments, adding the torsion to the regression model significantly improved the quality of fit, and resulted in positive $\triangle \mathrm{BIC}$ values, and the results of both tests were consistent segment by segment. In addition, we found that for the entire sample, $\triangle \mathrm{BIC}$ was significantly greater than zero (average $\Delta \mathrm{BIC}=164.509$, $t_{4935}=73.092, p<0.001$ ). Thus, we conclude that, for the three-dimensional movements that we analyzed, the speed-curvature-torsion power law describes the data better than the speed-curvature power law.

Overall, the suturing movements from both datasets are described very well with the speed-curvature-torsion power law. This is depicted in Fig. 4 that presents data from one segment that was analyzed in our study. Figure 4(a) describes the speed $v$, curvature $\kappa$, and torsion $\tau$ of the movement as a function of time. This graph demonstrates the inverse relation between the speed and the curvature, and the weaker inverse relation between the speed and the torsion. For example, the gray dashed lines in the plot highlight the local minima of the speed that are located in the same locations as the local maxima of the curvature. Similar effects happen also in the local maxima of the torsion, but they are masked in this plot
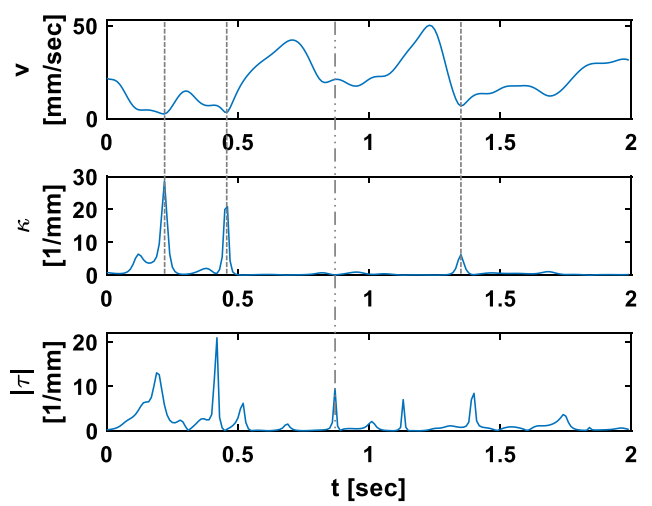

(a)

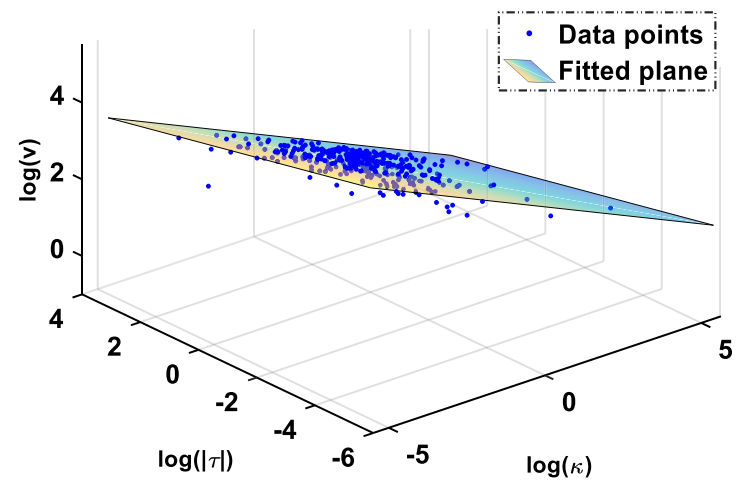

(b)

Fig. 4. An example from the data - segment (II) (insertion) of a nonmedical user from the dVRK-Open dataset. (a) The speed $v$, curvature $\kappa$, and torsion $\tau$ of the movement as a function of time. The gray dashed lines in the plot highlight the local maxima of the curvature, and the gray dash-dot line highlights the a local maximum of the torsion. (b) The relationship between $\log (v), \log (\kappa)$, and $\log (|\tau|)$. 
Table 1. Fitting the speed-curvature-torsion power-law.

\begin{tabular}{lccc}
\hline & $\beta$ & $\gamma$ & $R^{2}$ \\
\hline Open (dVRK-Open) & $-0.388 \pm 0.074$ & $-0.196 \pm 0.066$ & $0.844 \pm 0.102$ \\
dVRK (dVRK-Open) & $-0.401 \pm 0.072$ & $-0.198 \pm 0.053$ & $0.824 \pm 0.103$ \\
dVSS (JIGSAWS) & $-0.423 \pm 0.056$ & $-0.199 \pm 0.041$ & $0.870 \pm 0.080$ \\
\hline
\end{tabular}

Note: All numerical values are mean \pm 1 standard deviation.

by the effect of the curvature that has a larger power function. In addition, Fig. 4(b) illustrates the relationship between $\log (v), \log (\kappa)$, and $\log (|\tau|)$. The participant's movement in this example is in accordance with the speed-curvature-torsion power law, as evident in the homogeneous spread of the data points around the fitted plane. This example is in agreement with the quantitative analysis that is summarized in Table 1 . The overall goodness of fit of the movements to the power law is high $\left(R^{2}>0.82\right)$, and the average values of the curvature exponent $\beta$ and the torsion exponent $\gamma$ are close to the values of the exponents of the one-sixth power law. In addition, the values of $\beta$ and $\gamma$ for dVRK and dVSS are similar, and slightly different from the open data.

\section{1. dVRK-Open results}

The results of the statistical analysis we performed for this dataset are summarized in Table 2 and Fig. 5. The first effect that we investigated was expertise level. We found that the effect of expertise was statistically significant only for the speed gain factor $\alpha-$ it was larger

Table 2. Statistical analysis summary: dVRK-Open.

\begin{tabular}{lcrcr}
\hline Factor & Stat & $\alpha$ & \multicolumn{1}{c}{$\beta$} & $\gamma$ \\
\hline Expertise & $F_{1,12}$ & 7.435 & 4.141 & 0.022 \\
& $p$ & $\mathbf{0 . 0 1 8}$ & 0.065 & 0.884 \\
Teleoperation & $F_{1,12}$ & 301.355 & 8.514 & 0.041 \\
& $p$ & $<\mathbf{0 . 0 0 1}$ & $\mathbf{0 . 0 1 3}$ & 0.842 \\
Segment & $F_{1,12}$ & 14.188 & 47.68 & 223.254 \\
& $p$ & $\mathbf{0 . 0 0 3}$ & $<\mathbf{0 . 0 0 1}$ & $<\mathbf{0 . 0 0 1}$ \\
Expertise $*$ Teleoperation & $F_{1,12}$ & 5.707 & 0.211 & 0.003 \\
& $p$ & $\mathbf{0 . 0 3 4}$ & 0.654 & 0.959 \\
Expertise $*$ Segment & $F_{1,12}$ & 3.174 & 1.495 & 0.875 \\
Teleoperation*Segment & $p$ & 0.100 & 0.245 & 0.368 \\
& $F_{1,12}$ & 22.228 & 1.879 & 29.028 \\
Expertise $*$ Teleoperation $*$ & $F_{1,12}$ & 7.859 & 0.678 & 0.156 \\
Segment & & & & \\
& $p$ & $\mathbf{0 . 0 1 6}$ & 0.426 & 0.700 \\
\hline
\end{tabular}

Note: Three-way mixed model ANOVA. Bold font indicates statistically significant effects. for the experienced surgeons compared to nonmedical users (Fig. 5(a)). In addition, there was a statistically significant interaction between expertise and teleoperation condition. A post-hoc analysis revealed that the difference between $\alpha$ values of experienced surgeons and nonmedical users in the open condition was bigger than in the teleoperated condition $\left(\Delta \alpha=3.246, t_{12}=2.703\right.$, $p_{B}=0.038$; and $\Delta \alpha=1.280, t_{12}=2.443, p_{B}=0.062$, respectively). In addition, the second-order interaction between expertise, teleoperation, and segment was significant. This means that the interaction between teleoperation and expertise was more prominent in segment (II) than in segment (I), and further highlights the need for segmentation of surgical data.

The second effect was teleoperation condition. We found that the speed gain factor $\alpha$ and the curvature exponent $\beta$ were both statistically-significantly smaller in the teleoperated compared to the open conditions (Figs. 5(a) and 5(b)). For the torsion exponent $\gamma$, this difference was not statistically significant, but the interaction between teleoperation condition and segment was statistically significant (Fig. 5(c)). A post-hoc analysis revealed that in segment (I) (transport), the value of $\gamma$ in the teleoperated condition was not significantly different than in the open condition $\left(\Delta \gamma=0.021, t_{12}=2.237\right.$, $p_{B}=0.090$ ). However, in segment (II) (insertion) the value of $\gamma$ in the teleoperated condition was significantly lower than in the open condition $(\Delta \gamma=-0.024$, $\left.t_{12}=5.311, p_{B}<0.001\right)$. These results show that teleoperation affected the movements of the user, and that the effect depends on the segment of movement.

The last effect for this dataset was movement segment. We found that there were statistically significant differences between the two segments for all the three parameters $-\alpha, \beta$, and $\gamma$ (Figs. 5(a)-5(c)). In addition, for the speed gain factor $\alpha$, there was statistically significant interaction between segment and teleoperation condition. A post-hoc analysis revealed that the difference between the segments in the open condition was larger than the difference in the teleoperated condition $\left(\Delta \alpha=1.568, t_{12}=4.346, p_{B}=0.002 ;\right.$ and $\Delta \alpha=0.696$, $t_{12}=2.678, p_{B}=0.040$, respectively). These results suggest that the values of the power-law exponents and the speed gain factor may be used for building a segmentation algorithm for complex surgical tasks. 


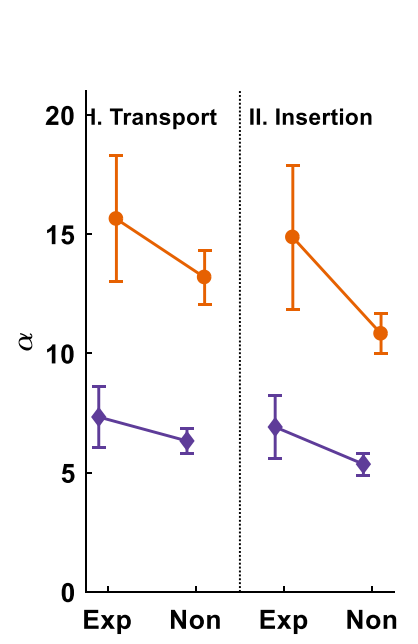

(a)

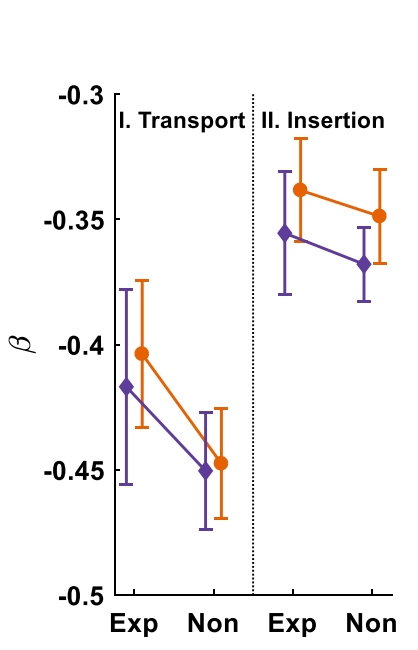

(b)

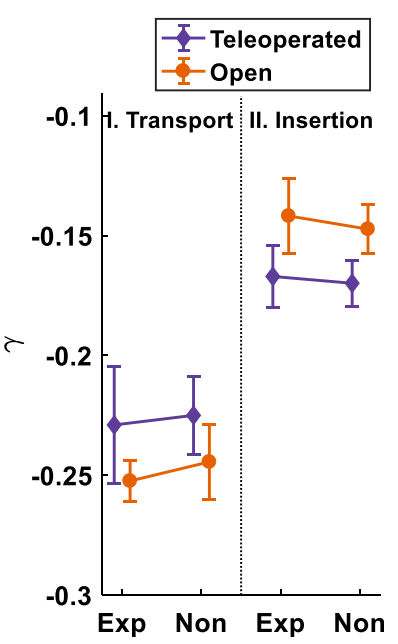

(c)

Fig. 5. The effects of expertise, teleoperation, and movement segment on the power-law parameters in the dVRK-Open dataset. The values of (a) the speed gain factor $\alpha$, (b) the curvature exponent $\beta$, and (c) torsion exponent $\gamma$ of experienced surgeons (Exp) and nonmedical users (Non) in the teleoperated and the open conditions for each segment. Markers are means, error bars are $95 \%$ confidence intervals.

\subsection{JIGSAWS results}

To evaluate the effect of expertise on the parameters of the power law, we performed regression analysis of the parameters as a function of GRS. The results of this analysis are depicted in Table 3 and Fig. 6 . The data of each surgeon is denoted by a distinctive color and marker. Our first question of interest focused on the global dependence of the power-law parameters on GRS. To appreciate these effects, we looked at all these data points together ignoring the information about the surgeon's identity. The summary of this analysis is the black dashed lines and the gray $95 \%$ confidence interval areas. There was no statistically significant global effect of expertise level (as measured by GRS) on the

Table 3. Statistical analysis summary: JIGSAWS.

\begin{tabular}{lcccc}
\hline Model & Stat & $\alpha$ & $\beta$ & $\gamma$ \\
\hline Expertise & & & & \\
$\quad$ Regression & slope & 0.032 & -0.001 & 0 \\
& $t_{37}$ & 1.459 & -2.582 & -0.349 \\
& $p$ & 0.153 & $\mathbf{0 . 0 1 4}$ & 0.729 \\
Repeated-measures & slope & -0.008 & 0 & 0.001 \\
regression & $F_{1,30}$ & 0.201 & 0.067 & 1.662 \\
& $p$ & 0.658 & 0.798 & 0.207 \\
Segment & & & & \\
$\quad$ Repeated-measures & $F_{3,21}$ & 37.284 & 32.603 & 31.230 \\
ANOVA & $p$ & $<\mathbf{0 . 0 0 1}$ & $<\mathbf{0 . 0 0 1}$ & $<\mathbf{0 . 0 0 1}$ \\
\hline
\end{tabular}

Note: Bold font indicates statistically significant effects. parameters $\alpha$ and $\gamma$. However, expertise level had significant global effect on the parameter $\beta$ : it decreased as the GRS increased.

Our second question of interest was whether within the different trials of each surgeon, there is an effect of GRS on the power-law parameters. The summary of this analysis is highlighted in the colored lines in Fig. 6. These lines were fitted with a repeated-measures regression model, which yields a single slope estimation, but each participant has a different intercept (and therefore, there are eight different colored lines with identical slopes in each panel). The results of this analysis show that the GRS of each individual trial had no significant effect on the distribution of values of all the speed-curvaturetorsion power-law parameters $(\alpha, \beta$, and $\gamma)$. This together with the previous result suggests that the global dependency of $\beta$ on GRS was due to difference between the surgeons, and that the parameter $\beta$ can be useful for classification of surgical expertise, but not of the quality of the individual movement.

Our last question was how movement segment affects the parameters of the power law. Figure 7 depicts the values of the power law in the four segments of the suturing task. A prominent observation from Fig. 7 is the big differences between the segments. The results of repeated-measures model ANOVA support this observation, and show that there were statistically significant differences between the segments for all the three parameters $-\alpha, \beta$, and $\gamma$. These results suggest that the values of the power law may be useful for segmentation of complex surgical tasks. 


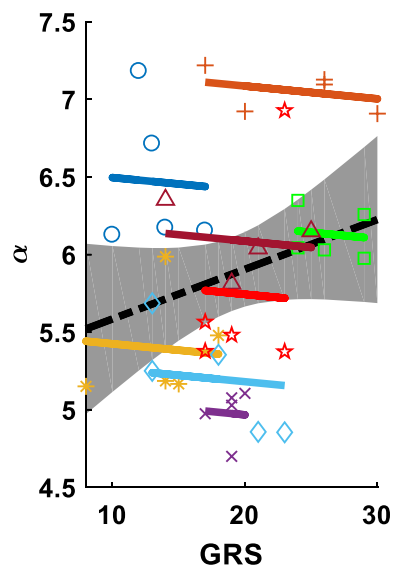

(a)

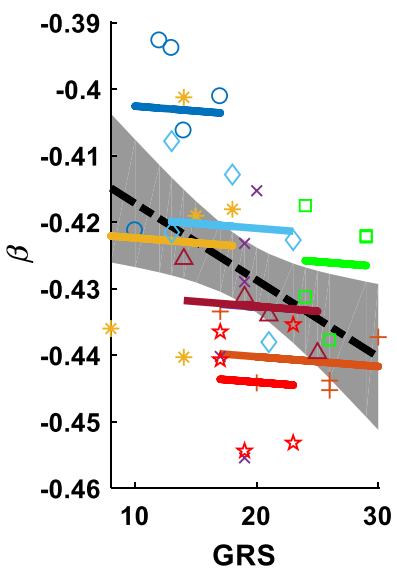

(b)

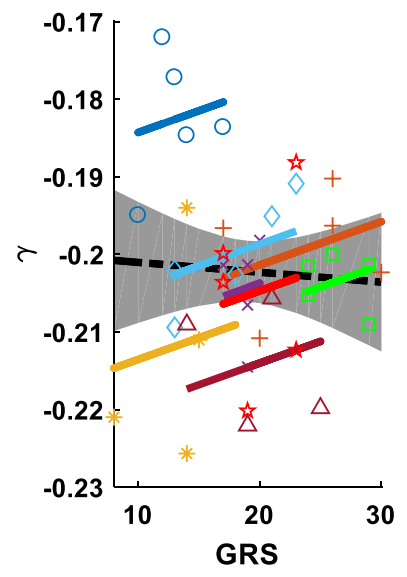

(c)

Fig. 6. The dependence of the power-law parameters on the level of expertise as evaluated with GRS in the JIGSAWS dataset. The values of (a) the speed gain factor $\alpha$, (b) the curvature exponent $\beta$, and (c) torsion exponent $\gamma$ of each trial are depicted as a function of expertise level (GRS). The different colors and markers indicate the eight participants. The colored lines represent the fit of the repeated-measures regression model, depicting an individual regression line for each participant. The black dashed lines are the global linear regression lines, and the gray areas around them are 95\% confidence interval areas of the global regression lines. This global regression depicts the average dependency of the parameter on GRS without taking into account the individual participants' differences.

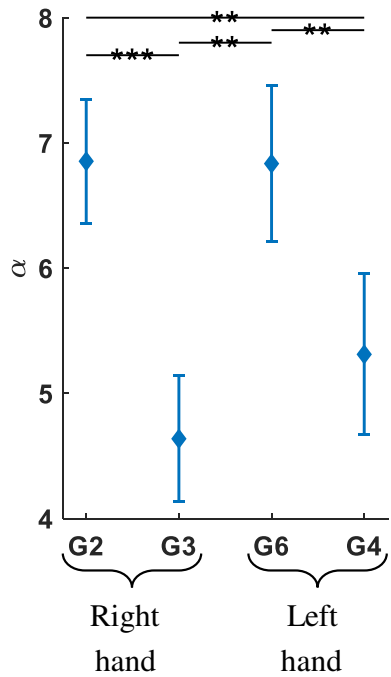

(a)

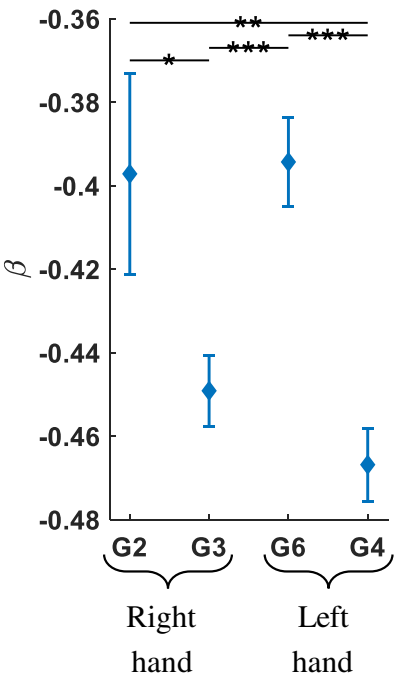

(b)

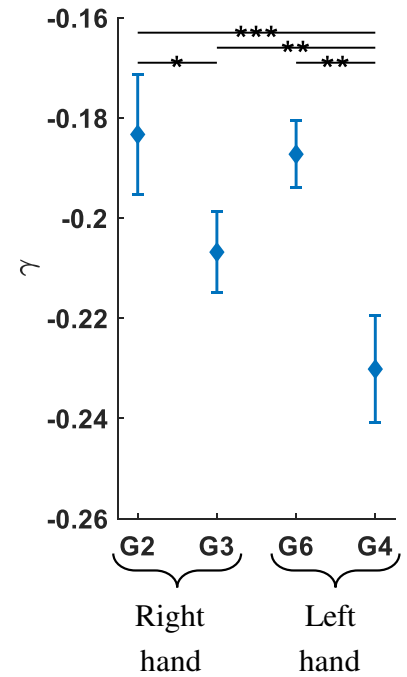

(c)

Fig. 7. The effect of movement segment on the power-law parameters in the JIGSAWS dataset. The values of (a) the speed gain factor $\alpha$, (b) the curvature exponent $\beta$, and (c) torsion exponent $\gamma$ for each segment: (G2) positioning, (G3) pushing, (G6) pulling, and (G4) transferring. Markers are means, error bars are $95 \%$ confidence intervals. ${ }^{*} p_{B}<0.05,{ }^{* *} p_{B}<0.01$, and ${ }^{* * *} p_{B}<0.001$.

\section{Discussion}

In this study, we characterized teleoperated and open suturing movements that were performed by participants with different levels of surgical skill. We analyzed the relationship between the speed of the suturing movements and their geometry - path curvature and torsion - in data from two different datasets. We found that the three-dimensional speed-curvature-torsion power law describes the suturing movements better than the planar speed-curvature power law. In addition, we found that expertise level, teleoperation, and movement segment affect this relationship.

\subsection{Comparing the power laws}

The fit of the speed-curvature-torsion power law was very good compared to the typical goodness of fit in such analyses [45, 46] supporting the assertion that indeed 
there is a characteristic relationship between the speed of the movements and their path curvature and torsion. Moreover, our model comparison analysis highlighted the importance of including the torsion in the model. This suggests that while the speed-curvature power law is a good first-order approximation for extracting important attributes of surgical motion $[43,44]$, in the tasks that we analyzed, significant amount of the variability in speed can be explained with torsion. This suggests that using the three-dimensional model, we are more likely to obtain a better dissection of the contribution of the speed gain factor and the individual exponents. Therefore, in analysis of tasks with movements that deviate from planarity, the speed-curvature-torsion power law should be used.

\subsection{Surgical expertise}

Our results suggest that the experience of a surgeon affects the parameters of the speed-curvature-torsion power law. This finding is in agreement with a previous study that reported that the speed gain factor $\alpha$ from the two-thirds power law can be useful for predicting surgical skill [44]. In our analysis of the dVRK-Open dataset, we found the speed gain factor $\alpha$ of experienced surgeons was larger than that of nonmedical users, but the effect was much more pronounced in the open condition (and not significant in the teleoperated condition). It is possible that this difference was a result of the dVRK teleoperation being not sufficiently similar to the clinical system that the experienced surgeons are using. Moreover, in the JIGSAWS dataset $\alpha$ increased with GRS (expertise level), albeit the effect was not significant. In this dataset, all the participants had surgical experience, and therefore, it is likely that the difference between their expertise levels was relatively small.

The speed gain factor $\alpha$ is related to overall tempo of motion [55]. Hence, the effect of expertise level on $\alpha$ may be related to the well-documented differences between completion times of participants with different surgical skill levels [20, 21, 23]. In addition, the planar two-thirds power law can be predicted by smoothness of movement maximization [64], and hence, the relation between expertise and the parameters of the power law may also be related to the smoothness of movement that is characteristic of skilled performance $[25,26]$.

It is important to note that fitting $\alpha$ together with $\beta$ and $\gamma$ as we did in this study, leads to dependence of the units of $\alpha$ on the fitted value of $\beta$ and $\gamma$. It also leads to sensitivity of $\alpha$ to the units used for calculations. Hence, future work is needed to investigate whether a metric for surgical expertise level that will be based on the speed gain factor $\alpha$, as we calculated it, will be successful in classifying between expertise levels.

The value of the exponent $\beta$ in JIGSAWS decreased as the surgical skill (GRS) increased. This results suggests that $\beta$ may be used as an objective metric for surgical skill. However, this hypothesis should be explored in depth, with a greater number of movements and participants. For example, even in our study here, in the dVRK-Open dataset, the difference between $\beta$ values of experienced surgeons and nonmedical users was not statistically significant, and seems to be reversed: experienced surgeons had higher $\beta$ than the nonmedical users. A possible explanation for the discrepancy between the results of the two datasets is the differences in ages between the participants [65]. The nonmedical users in dVRK-Open were graduate students, and hence were much younger than the experienced surgeons. Thus, the results in dVRK-Open dataset may be due to the combined effect of surgical expertise and differences in age. Note that the age contrasts here are between engineering students aged 21-25 and experienced surgeons all aged over 39, and not between children and adults as in [49]. Furthermore, while we do not have access to the ages of the surgeons in the JIGSAWS dataset, expertise and age within the dataset may be correlated. An experiment with age-matched nonmedical users and experienced surgeons can isolate the origin of the discrepancies in future studies.

In addition, the differences in results between the two datasets may be also related to substantial procedural differences between them. First, the tissue used for the task was different: in the dVRK-Open dataset, the tissue was made of foam, and in the JIGSAWS the tissue was made of elastic fabric. Second, both segments in the dVRK-Open dataset were performed using one hand, whereas in the JIGSAWS datasets, the suturing was performed using both hands.

\subsection{Teleoperation}

The results from the dVRK-Open dataset show that using a robot-assisted surgery system to perform the suturing task significantly affected the speed-curvature-torsion power-law parameters. The difference between the speed gain factor $\alpha$ values from the teleoperated and the open sessions is likely due to the fact that we analyzed the data from the PSMs. To control the PSM, the speed of the Master Tool Manipulator (MTM) was downscaled by a factor of 3. Because the speed gain factor $\alpha$ is related to overall tempo of motion [55], the difference in $\alpha$ values between the conditions is likely a result of this downscaling. Our hypothesis was that the dynamic constraints in the teleoperation movements will affect the speedcurvature-torsion power-law parameters. Indeed, in the dVRK-Open dataset the curvature exponent $\beta$ differed between the teleoperated and the open conditions. In addition, the average $\beta$ values that were obtained from the JIGSAWS dataset, in which all the movements were teleoperated, and the teleoperated condition in dVRK-Open dataset were similar. These results are consistent with the 
findings in [56], which suggested that dynamics can affect the relationship between the speed and curvature in planar movements.

In dVRK-Open, for the torsion exponent $\gamma$, the effect of teleoperation depended on the segment. This result may be due to the different nature of the two segments: segment (I), (transport) was a movement in the air, whereas segment (II) (insertion) included interaction with tissue. In segment (I), the movement was not likely affected by the absence of haptic feedback in the teleoperated condition, and thus, in segment (I) the difference between teleoperated and open movements was small and not significant. On the other hand, in segment (II), there was interaction with tissue, the movement was probably affected by haptic feedback, and therefore, the difference between teleoperated and open movements was greater. Further investigation of these findings is needed to examine whether this result was indeed caused by the haptic feedback.

Using the speed-curvature-torsion power law and other invariants from computational motor control may help to improve the design of surgical robots. For example, comparing the parameters of the three-dimensional power law in open and teleoperated movements can reveal differences in the way we move using teleoperation that we cannot reveal using other measures. Our results show that the use of the surgical robot affects the parameters of the power law. This adds to the previously reported results of effects of robotic manipulators on movements of surgeons [34, 35]. Understanding these differences may help to design robots that feel more natural and intuitive, and then use the parameters of the power law as a possible benchmark for a successful design.

Robotic assistance systems are developed today to help surgeons to perform and learn surgical tasks. For example, virtual fixtures are used to help surgeons to move the tools along specific path [66-69], or influencing the learning processes by applying forces that push the surgeon away from desired path or back to the desired path during training [70]. In [71], participants applied lower force when they held an end-effector that moved in accordance with the two-thirds power law comparing to an end-effector that moved not according to the power law. Their result suggests that for easy and intuitive assistance, it may be helpful to design shared control [72] and assistive systems in a way that is compatible with the natural movement of humans. These results together with our characterization of surgical suturing suggest that virtual fixtures that enforce trajectories may be better if they adhere to the speed-curvature-torsion power law. Importantly, the specific parameters of the power law will have to be determined as part of the controller design, or alternatively, may be left as a tuning parameter for the user. This framework can also be relevant to other applications of teleoperation [73-75], shared control [76, 77], devices such as exoskeletons [78] and prostheses, and even trajectory generation for autonomous systems $[79,80]$.

\subsection{Movement segment}

We found that all the power-law parameters $(\alpha, \beta$, and $\gamma)$ were affected by movement segment. These results were obtained from both datasets. In dVRK-Open, the movements were performed using one hand, and we investigated two segments. In JIGSAWS, we investigated four segments - two segments performed using the right hand and two using the left hand. Our results showed that for each power-law parameter, the differences between segments that performed using the same hand [(I) transport-(II) insertion, (G2) positioning-(G3) pushing, and (G6) pulling-(G4) transferring] were significant. These differences suggest that power-law parameters may be used for development of automatic segmentation algorithms. In this study, we calculated the power-law parameters on segments that were already set. Previous studies suggested that the speed gain factor $\alpha$ can be used for segmentation of surgical task $[43,58]$. In future studies, we will develop and examine a segmentation algorithm that will divide surgical task into small segments based on more parameters of the power law ( $\beta$ and $\gamma$ ). We will compare our algorithm to other algorithms for segmentation of surgical tasks [37-40].

There is a similarity between the segments from dVRK-Open and JIGSAWS: (I) transport and (G2) positioning, (II) insertion and (G3) pushing. Despite these similarities, it seems that for the parameters $\beta$ and $\gamma$, the difference between (I) transport and (II) insertion (Fig. 5) is not in the same direction as the difference between (G2) positioning and (G3) pushing (Fig. 7). However, a careful examination suggests that the values of $\beta$ and $\gamma$ that are obtained from JIGSAWS, are all in a similar range as those parameters in segment (I) from dVRK-Open. As noted earlier, dynamic factors can affect the parameters of the power law [56]. The tissues used in the experiments carried out to collect the two datasets were different. The tissue in dVRK-Open was made of foam, while in JIGSAWS, the tissue is made of an elastic fabric. Pushing the needle through the foam in dVRKOpen is very different from pushing a needle in the air, but pushing a needle through the elastic fabric may be more similar to pushing a needle in the air. Thus, in dVRK-Open, the values in segment (II) were higher than in segment (I), and in JIGSAWS, the values in (G3) were lower than in (G2), but still within the range of the values of moment in the air. This explanation can be explored in future studies by analyzing movements that will interact with tissues of various properties.

A segmentation algorithm that may be developed using the speed-curvature-torsion power-law parameters will highlight segmentation based on motor 
primitives [41] rather than to procedural segments. Such kinematic segmentation may compliment the large body of literature on surgical task segmentation using contextual information [37-40]. Combining these approaches with the motor primitives may lead to the development of even more successful algorithms for the important task of surgical movement segmentation.

In computational motor control, there is a debate whether indeed segmented kinematics reflect segmented control strategies [55, 81]. However, there is increased body of evidence supporting segmented or intermittent control [2, 82-84], and movement chunking [85]. Certainly, the debate about the neural origin of this segmentation does not impede the utility of our proposed analysis in understanding of surgical movements.

\subsection{Limitations}

Based on the presented results, we are convinced that our new approach to characterization of surgical motion can help to quantify expertise, improve teleoperation, and improve task segmentation algorithms. However, there are also several limitations in our study.

In the dVRK-Open, the participants were nonmedical users and experienced surgeons. To understand whether the parameters can be useful for distinguishing between more similar skill levels, an experiment could be conducted in which medical trainees will also participate. Our rationale in choosing the engineering students in this first study was to use two very different groups maximizing the possible differences in expertise comparison between participants without any experience and participants with experience. This problem is partially mitigated in the JIGSAWS dataset, but the sample size is small. In future studies, we plan to use our new approach to look for difference between surgical residents and experienced surgeons, and between experienced surgeons with much larger span of case experience and skill.

To acquire the data in the open condition of the dVRKOpen dataset, we used magnetic pose trackers. The position of the trackers was selected to minimize signal distortion and noise due to the needle driver. To identify possible distortions, we inspected all the paths visually. To mitigate noise effects, we low-pass filtered the data. Nevertheless, the open condition data is still likely affected by magnetic tracking artifacts, and it would be valuable to verify our results in future studies with other motion tracking methods.

We presented an analysis of the first two segments from the suturing task of the dVRK-Open dataset. Because there are no video recordings of the movements, we could not perform a reliable segmentation of the last two segments of the movement. These last two segments would likely reveal additional insights of motion characteristics that could be indicative of skill, and could reveal additional differences between teleoperated and open suturing. However, segment (II) involves insertion of the needle through tissue, which requires rotation of the tool. This action is not simple for inexperienced participants, and therefore we believe that even the first two segments can be indicative of skill. Moreover, segmentation of the last two segments could have helped in comparing between the datasets. We chose to use both datasets because they have several similar aspects for comparison. In addition, each dataset has its own unique features. The dVRK-Open dataset allows for the comparison of open and teleoperated suturing, and the JIGSAWS datasets allows for comparison of movements based on their objective skill level scores.

Segment (I) and segment (II) of the dVRK-Open dataset are in air and tissue, respectively. From our results we cannot be sure that power-law parameters are able to differentiate between tasks, not just between motions in different materials. However, even if the parameters can only distinguish between motions in different materials, they can be useful as part of the segmentation algorithm of surgical movements, to distinguish between segments with and without contact with tissue. In addition, we analyzed in this paper movements with relatively small variations between participants. To examine whether the power-law parameters will be useful for automated segmentation algorithm, future studies will be focused on analyzing less structured movements.

\section{Conclusion}

We present a novel characterization of surgical movements using the speed-curvature-torsion power law. We found that expertise, teleoperation, and task segment significantly affected the gain and exponents of the power law. This opens a promising avenue for improving the evaluation of surgical skill, teleoperation control design, and surgical gestures segmentation.

\section{Acknowledgments}

We thank Allison Okamura, Michael Hsieh, Zhan Fan Quek, and Yuhang Che, for their help in obtaining the experimental data. We thank Tamar Flash for useful discussions on the speed-curvature-torsion power law. This study was supported in part by the Helmsley Charitable Trust through the Agricultural, Biological and Cognitive Robotics Initiative and by the Marcus Endowment Fund both at Ben-Gurion University of the Negev and the ISF Grant No. 823/15. Yarden Sharon was supported by the Besor Fellowship. 


\section{References}

1. D. M. Wolpert, Z. Ghahramani and M. I. Jordan, An internal model for sensorimotor integration, Science 269(5232) (1995) 1880-1882.

2. A. Karniel, Open questions in computational motor control, J. Integr. Neurosci. 10(3) (2011) 385-411.

3. S. Schaal and N. Schweighofer, Computational motor control in humans and robots, Curr. Opin. Neurobiol. 15(6) (2005) 675-682.

4. M. Kawato, Internal models for motor control and trajectory planning, Curr. Opin. Neurobiol. 9(6) (1999) 718-727.

5. J. W. Krakauer and P. Mazzoni, Human sensorimotor learning: Adaptation, skill, and beyond, Curr. Opin. Neurobiol. 21(4) (2011) 636-644.

6. R. Shadmehr and S. Mussa-Ivaldi, Biological Learning and Control: How the Brain Builds Representations, Predicts Events, and Makes Decisions (MIT Press, Cambridge, 2012).

7. A. M. Jarc and I. Nisky, Robot-assisted surgery: An emerging platform for human neuroscience research, Front. Hum. Neurosci. 9 (2015) 315.

8. J. D. Birkmeyer, J. F. Finks, A. O'Reilly, M. Oerline, A. M. Carlin, A. R. Nunn, J. Dimick, M. Banerjee and N. J. Birkmeyer, Surgical skill and complication rates after bariatric surgery, N. Engl. J. Med. 369(15) (2013) 1434-1442.

9. K. A. Ericsson, Deliberate practice and the acquisition and maintenance of expert performance in medicine and related domains, Acad. Med. 79(10) (2004) S70-S81.

10. R. K. Reznick and H. MacRae, Teaching surgical skills: Changes in the wind, N. Engl. J. Med. 355(25) (2006) 2664-2669.

11. M. C. Vassiliou, L. S. Feldman, C. G. Andrew, S. Bergman, K. Leffondr, D. Stanbridge and G. M. Fried, A global assessment tool for evaluation of intraoperative laparoscopic skills, Am. J. Surg. 190(1) (2005) 107-113.

12. J. Martin, G. Regehr, R. Reznick, H. MacRae, J. Murnaghan, C. Hutchison and M. Brown, Objective structured assessment of technical skill (osats) for surgical residents, Br. J. Surg. 84(2) (1997) 273-278.

13. V. Datta, S. Mackay, M. Mandalia and A. Darzi, The use of electromagnetic motion tracking analysis to objectively measure open surgical skill in the laboratory-based model, J. Am. Coll. Surg. 193 (5) (2001) 479-485.

14. M. K. Chmarra, N. H. Bakker, C. A. Grimbergen and J. Dankelman, TrEndo, a device for tracking minimally invasive surgical instruments in training setups, Sens. Actuators A, Phys. 126(2) (2006) 328-334.

15. M. S. Wilson, A. Middlebrook, C. Sutton, R. Stone and R. F. McCloy, MIST VR: A virtual reality trainer for laparoscopic surgery assesses performance, Ann. R. Coll. Surg. Engl. 79(6) (1997) 403-404.

16. J. Eriksen and T. Grantcharov, Objective assessment of laparoscopic skills using a virtual reality stimulator, Surg. Endosc. 19(9) (2005) 1216-1219.

17. G. S. Guthart and J. K. Salisbury, The intuitive ${ }^{\mathrm{TM}}$ telesurgery system: Overview and application, in Proc. IEEE Int. Conf. Robotics and Automation (2000), pp. 618-621.

18. P. Kazanzidesf, Z. Chen, A. Deguet, G. S. Fischer, R. H. Taylor and S. P. DiMaio, An open-source research kit for the da Vinci surgical system, in Proc. IEEE Int. Conf. Robotics and Automation (2014), pp. 6434-6439.

19. A. Van Rij, J. McDonald, R. Pettigrew, M. Putterill, C. Reddy and J. Wright, Cusum as an aid to early assessment of the surgical trainee, Br. J. Surg. 82(11) (1995) 1500-1503.

20. T. J. Tausch, T. M. Kowalewski, L. W. White, P. S. McDonough, T. C. Brand and T. S. Lendvay, Content and construct validation of a robotic surgery curriculum using an electromagnetic instrument tracker, J. Urol. 188(3) (2012) 919-923.

21. N. Rittenhouse, B. Sharma, R. Sonnadara, A. Mihailidis and T. Grantcharov, Design and validation of an assessment tool for open surgical procedures, Surg. Endosc. 28(3) (2014) 918-924.
22. P. A. Kenney, M. F. Wszolek, J. J. Gould, J. A. Libertino and A. Moinzadeh, Face, content, and construct validity of DV-trainer, a novel virtual reality simulator for robotic surgery, Urology 73(6) (2009) 1288-1292.

23. E. F. Hofstad, C. Vapenstad, M. K. Chmarra, T. Langø, E. Kuhry and R. Märvik, A study of psychomotor skills in minimally invasive surgery: What differentiates expert and nonexpert performance, Surg. Endosc. 27(3) (2013) 854-863.

24. K. Van Sickle, D. MeClusky III, A. Gallagher and C. Smith, Construct validation of the promis simulator using a novel laparoscopic suturing task, Surg. Endosc. 19(9) (2005) 1227-1231.

25. S. Estrada, C. Duran, D. Schulz, J. Bismuth, M. D. Byrne and M. K. O'Malley, Smoothness of surgical tool tip motion correlates to skill in endovascular tasks, IEEE Trans. Hum.-Mach. Syst. 46(5) (2016) 647-659.

26. K. Liang, Y. Xing, J. Li, S. Wang, A. Li and J. Li, Motion control skill assessment based on kinematic analysis of robotic end-effector movements, Int. J. Med. Robot. Comput. Assist. Surg. 14(1) (2017) e1845.

27. B. Poursartip, M.-E. LeBel, L. C. McCracken, A. Escoto, R. V. Patel, M. D. Naish and A. L. Trejos, Energy-based metrics for arthroscopic skills assessment, Sensors 17(8) (2017) 1808.

28. B. Poursartip, M. E. LeBel, R. Patel, M. Naish and A. L. Trejos, Analysis of energy-based metrics for laparoscopic skills assessment, IEEE Trans. Biomed. Eng. (2017), doi: 10.1109/TBME. 2017.2706499.

29. J. D. Brown, C. E. O'Brien, S. C. Leung, K. R. Dumon, D. I. Lee and K. J. Kuchenbecker, Using contact forces and robot arm accelerations to automatically rate surgeon skill at peg transfer, IEEE Trans. Biomed. Eng. 64(9) (2017) 2263-2275.

30. S. Maeso, M. Reza, J. A. Mayol, J. A. Blasco, M. Guerra, E. Andradas and M. N. Plana, Efficacy of the da Vinci surgical system in abdominal surgery compared with that of laparoscopy: A systematic review and meta-analysis, Ann. Surg. 252(2) (2010) 254-262.

31. K. Moorthy, Y. Munz, A. Dosis, J. Hernandez, S. Martin, F. Bello, T. Rockall and A. Darzi, Dexterity enhancement with robotic surgery, Surg. Endosc. 18(5) (2004) 790-795.

32. A. R. Lanfranco, A. E. Castellanos, J. P. Desai and W. C. Meyers, Robotic surgery: A current perspective, Ann. Surg. 239(1) (2004) $14-21$.

33. A. M. Okamura, Haptic feedback in robot-assisted minimally invasive surgery, Curr. Opin. Urol. 19(1) (2009) 102-107.

34. I. Nisky, M. H. Hsieh and A. M. Okamura, Uncontrolled manifold analysis of arm joint angle variability during robotic teleoperation and freehand movement of surgeons and novices, IEEE Trans. Biomed. Eng. 61(12) (2014) 2869-2881.

35. I. Nisky, A. M. Okamura and M. H. Hsieh, Effects of robotic manipulators on movements of novices and surgeons, Surg. Endosc. 28(7) (2014) 2145-2158.

36. H. C. Lin, I. Shafran, D. Yuh and G. D. Hager, Towards automatic skill evaluation: Detection and segmentation of robot-assisted surgical motions, Comput. Aided Surg. 11(5) (2006) 220-230.

37. A. James, D. Vieira, B. Lo, A. Darzi and G.-Z. Yang, Eye-gaze driven surgical workflow segmentation, in Proc. Int. Conf. Medical Image Computing and Computer-Assisted Intervention (2007), pp. 110-117.

38. L. Zappella, B. Béjar, G. Hager and R. Vidal, Surgical gesture classification from video and kinematic data, Med. Image Anal. 17(7) (2013) 732-745.

39. N. Ahmidi, L. Tao, S. Sefati, Y. Gao, C. Lea, B. B. Haro, L. Zappella, S. Khudanpur, R. Vidal and G. D. Hager, A dataset and benchmarks for segmentation and recognition of gestures in robotic surgery, IEEE Trans. Biomed. Eng. 64(9) (2017) 2025-2041.

40. F. Despinoy, D. Bouget, G. Forestier, C. Penet, N. Zemiti, P. Poignet and P. Jannin, Unsupervised trajectory segmentation for surgical gesture recognition in robotic training, IEEE Trans. Biomed. Eng. 63(6) (2016) 1280-1291. 
41. P. Viviani and M. Cenzato, Segmentation and coupling in complex movements, J. Exp. Psychol., Hum. Percept. Perform. 11(6) (1985) 828-845.

42. F. Lacquaniti, C. Terzuolo and P. Viviani, The law relating the kinematic and figural aspects of drawing movements, Acta Psychol. 54(1) (1983) 115-130.

43. S. B. Shafiei, K. A. Guru and E. T. Esfahani, Using two-third power law for segmentation of hand movement in robotic assisted surgery, in Proc. AMSE 2015 Int. Design Engineering Technical Conf. and Computers and Information in Engineering Conf. (2015).

44. A. French, T. S. Lendvay, R. M. Sweet and T. M. Kowalewski, Predicting surgical skill from the first $\mathrm{n}$ seconds of a task: Value over task time using the isogony principle, Int. J. Comput. Assist. Radiol. Surg. 12(7) (2017) 1161-1170.

45. F. E. Pollick, U. Maoz, A. A. Handzel, P. J. Giblin, G. Sapiro and T. Flash, Three-dimensional arm movements at constant equi-affine speed, Cortex 45(3) (2009) 325-339.

46. U. Maoz, A. Berthoz and T. Flash, Complex unconstrained threedimensional hand movement and constant equi-affine speed, J. Neurophysiol. 101(2) (2009) 1002-1015.

47. I. Nisky, Y. Che, Z. F. Quek, M. Weber, M. H. Hsieh and A. M. Okamura, Teleoperated versus open needle driving: Kinematic analysis of experienced surgeons and novice users, in Proc. IEEE Int. Conf. Robotics and Automation (2015) pp. 5371-5377.

48. Y. Gao, S. S. Vedula, C. E. Reiley, N. Ahmidi, B. Varadarajan, H. C. Lin, L. Tao, L. Zappella, B. Béjar and D. D. Yuh, The JHU-ISI gesture and skill assessment working set (JIGSAWS): A surgical activity dataset for human motion modeling, in Proc. MICCAI Workshop, Vol. 3, (2014).

49. P. Viviani and R. Schneider, A developmental study of the relationship between geometry and kinematics in drawing movements, J. Exp. Psychol., Hum. Percept. Perform. 17(1) (1991) 198-218.

50. C. de'Sperati and P. Viviani, The relationship between curvature and velocity in two-dimensional smooth pursuit eye movements, J. Neurosci. 17(10) (1997) 3932-3945.

51. S. Vieilledent, Y. Kerlirzin, S. Dalbera and A. Berthoz, Relationship between velocity and curvature of a human locomotor trajectory, Neurosci. Lett. 305(1) (2001) 65-69.

52. M. Karklinsky and T. Flash, Timing of continuous motor imagery: The two-thirds power law originates in trajectory planning, $J$. Neurophysiol. 113(7) (2015) 2490-2499.

53. S. Schaal and D. Sternad, Origins and violations of the $2 / 3$ power law in rhythmic three-dimensional arm movements, Exp. Brain Res. 136(1) (2001) 60-72.

54. D. Huh and T. J. Sejnowski, Spectrum of power laws for curved hand movements, Proc. Natl. Acad. Sci. USA 112(29) (2015) E3950-E3958.

55. M. J. Richardson and T. Flash, Comparing smooth arm movements with the two-thirds power law and the related segmented-control hypothesis, J. Neurosci. 22(18) (2002) 8201-8211.

56. G. Catavitello, Y. P. Ivanenko, F. Lacquaniti and P. Viviani, Drawing ellipses in water: Evidence for dynamic constraints in the relation between velocity and path curvature, Exp. Brain Res. 234(6) (2016) 1649-1657.

57. D. M. Endres, Y. Meirovitch, T. Flash and M. A. Giese, Segmenting sign language into motor primitives with Bayesian binning, Front. Comput. Neurosci. 7 (2013) 68.

58. J. Cifuentes, P. Boulanger, M. T. Pham, R. Moreau and F. Prieto, Automatic gesture analysis using constant affine velocity, in Proc. Annu. Int. Conf. IEEE Engineering in Medicine and Biology Society (2014), pp. 1826-1829.

59. Ascension Technology Corporation, $3 D$ Guidance trakSTAR ${ }^{\mathrm{TM}}$ Installation and Operation Guide (Ascension Technology Corp., 2009).
60. Y. Sharon, T. S. Lendvay and I. Nisky, Instrument orientation-based metrics for surgical skill evaluation in robot-assisted and open needle driving, arXiv:1709.09452 [CS.RO].

61. S. A. Glantz and B. K. Slinker, Primer of Applied Regression and Analysis of Variance, 2 edn. (McGraw-Hill Education, 2000).

62. G. Schwarz, Estimating the dimension of a model, Ann. Stat. 6(2) (1978) 461-464.

63. M. J. Fard, S. Ameri, R. D. Ellis, R. B. Chinnam, A. K. Pandya and M. D. Klein, Automated robotassisted surgical skill evaluation: Predictive analytics approach, Int. J. Med. Robot. Comput. Assist. Surg. 14(1) (2018) e1850.

64. T. Flash, Y. Meirovitch and A. Barliya, Models of human movement: Trajectory planning and inverse kinematics studies, Robot. Auton. Syst. 61(4) (2013) 330-339.

65. T. L. Gibo, A. J. Bastian and A. M. Okamura, Effect of age on stiffness modulation during postural maintenance of the arm, in Proc. IEEE Int. Conf. Rehabilitation Robotics (2013), pp. 1-6.

66. J. Abbott, P. Marayong and A. Okamura, Haptic virtual fixtures for robot-assisted manipulation, in Proc. 12th Int. Symp. Robotics Research, Springer Tracts in Advanced Robotics, Vol. 28 (2007), pp. 49-64.

67. S. A. Bowyer, B. L. Davies and Y. B. Rodriguez, Active constraints/ virtual fixtures: A survey, IEEE Trans. Robot. 30(1) (2014) 138157.

68. N. Enayati, E. D. Momi and G. Ferrigno, Haptics in robot-assisted surgery: Challenges and benefits, IEEE Rev. Biomed. Eng. 9 (2016) 49-65.

69. M. A. Vitrani, C. Poquet and G. Morel, Applying virtual fixtures to the distal end of a minimally invasive surgery instrument, IEEE Trans. Robot. 33(1) (2017) 114-123.

70. M. M. Coad, A. M. Okamura, S. Wren, Y. Mintz, T. S. Lendvay, A. M. Jarc and I. Nisky, Training in divergent and convergent force fields during 6-dof teleoperation with a robot-assisted surgical system, in proc. IEEE World Haptic. Conf. (2017), pp. 195-200.

71. P. Maurice, M. E. Huber, N. Hogan and D. Sternad, Velocity-curvature patterns limit human-robot physical interaction, IEEE Robot. Autom. Lett. 3(1) (2018) 249-256.

72. D. A. Abbink, M. Mulder and E. R. Boer, Haptic shared control: Smoothly shifting control authority?, Cogn. Technol. Work 14(1) (2012) 19-28.

73. N. Jarrass, J. Paik, V. Pasqui and G. Morel, How can human motion prediction increase transparency?, in Proc. IEEE Int. Conf. Robotics and Automation (2008), pp. 2134-2139.

74. I. Nisky, F. A. Mussa-Ivaldi and A. Karniel, Analytical study of perceptual and motor transparency in bilateral teleoperation, IEEE Trans. Hum.-Mach. Syst. 43(6) (2013) 570-582.

75. J. Buzzi, C. Gatti, G. Ferrigno and E. D. Momi, Analysis of joint and hand impedance during teleoperation and free-hand task execution, IEEE Robot. Autom. Lett. 2(3) (2017) 1733-1739.

76. H. Boessenkool, D. A. Abbink, C. J. M. Heemskerk, F. C. T. van der Helm and J. G. W. Wildenbeest, A task-specific analysis of the benefit of haptic shared control during telemanipulation, IEEE Trans. Haptics 6(1) (2013) 2-12.

77. J. Smisek, E. Sunil, M. M. van Paassen, D. A. Abbink and M. Mulder, Neuromuscular-system-based tuning of a haptic shared control interface for UAV teleoperation, IEEE Trans. Hum.-Mach. Syst. 47(4) (2017) 449-461.

78. N. Jarrasse and G. Morel, Connecting a human limb to an exoskeleton, IEEE Trans. Robot. 28(3) (2012) 697-709.

79. Y. Meirovitch, D. Bennequin and T. Flash, Geometrical invariance and smoothness maximization for task-space movement generation, IEEE Trans. Robot. 32(4) (2016) 837-853.

80. M. Karklinsky, M. Naveau, A. Mukovskiy, O. Stasse, T. Flash and P. Soueres, Robust human-inspired power law trajectories for humanoid HRP-2 robot, in Proc. IEEE Conf. Biomedical Robotics and Biomechatronics (2016), pp. 106-113. 
81. D. Sternad and S. Schaal, Segmentation of endpoint trajectories does not imply segmented control, Exp. Brain. Res. 124(1) (1999) 118-136.

82. R. Leib and A. Karniel, Minimum acceleration with constraints of center of mass: A unified model for arm movements and object manipulation, J. Neurophysiol. 108(6) (2012) 1646-1655.

83. S. Ben-Itzhak and A. Karniel, Minimum acceleration criterion with constraints implies bang-bang control as an underlying principle

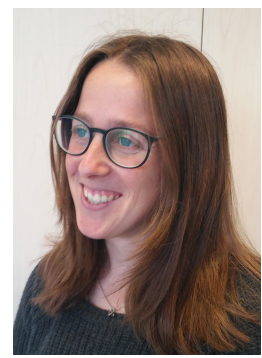

Yarden Sharon received her B.Sc. degree (magna cum laude) in Biomedical Engineering from the Department of Biomedical Engineering, Ben-Gurion University of the Negev, Israel, in 2016. She is currently a Ph.D. student in the Biomedical Robotics Lab at the Department of Biomedical Engineering, Ben-Gurion University of the Negev. She is a recipient of the Besor Fellowship for excellent Ph.D. students. Her research interests are surgical robotics, computational motor control, and surgical skill evaluation and acquisition.

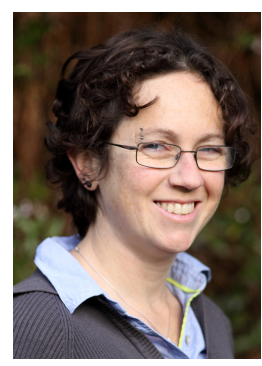

Ilana Nisky received the B.Sc. (summa cum laude), M.Sc. (summa cum laude), and Ph.D. degrees in Biomedical Engineering from the Department of Biomedical Engineering, BenGurion University of the Negev, Israel, in 2006, 2009 , and 2011, respectively. She is currently a Senior Lecturer in the Department of Biomedical Engineering, Ben-Gurion University of the Negev, where she is the Head of the Biomedical Robotics Lab. She was previously a Postdoctoral Research Fellow in the Department of Mechanical Engineering, Stanford University, USA. Her research interests include human motor control, haptics, robotics, human and machine learning, teleoperation, and robot-assisted surgery. She is a member of the BGU ABC Robotics Initiative and serves on the steering committee of the Zlotowski Center for Neuroscience. She is a member of IEEE, the Society for the Neural Control of Movement, the Society for Neuroscience, Technical Committee on Haptics, and an Executive Committee member of the Eurohaptics Society. for optimal trajectories of arm reaching movements, Neural Comput. 20(3) (2008) 779-812.

84. P. Gawthrop, I. Loram, M. Lakie and H. Gollee, Intermittent control: A computational theory of human control, Biol. Cybern. 104(1) (2011) 31-51.

85. P. Ramkumar, D. E. Acuna, M. Berniker, S. T. Grafton, R. S. Turner and K. P. Kording, Chunking as the result of an efficiency computation trade-off, Nat. Commun. 7 (2016) 12176. 